Proyecciones

Vol. 21, No 2, pp. 141-153, August 2002.

Universidad Católica del Norte

Antofagasta - Chile

DOI: 10.4067/S0716-09172002000200003

\title{
A NOTE ON ASYMPTOTIC SMOOTHNESS OF THE EXTENSIONS OF ZADEH
}

\author{
LAECIO C. BARROS \\ Universidade Estadual de Campinas - Brasil \\ SUZANA A. OLIVEIRA \\ Universidade de Sao Paulo - Brasil \\ and \\ PEDRO A. TONELLI \\ Universidade de Sao Paulo - Brasil
}

\begin{abstract}
The concept of asymptotic smooth transformation was introduced by J. Hale [10]. It is a very important property for a transformation between complete metric spaces to have a global attractor. This property has also consequences on asymptotic stability of attractors. In our work we study the conditions under which the Zadeh's extension of a continuous map $f: \mathbf{R}^{n} \rightarrow \mathbf{R}^{n}$ is asymptotically smooth in the complete metric space $\mathcal{F}\left(\mathbf{R}^{n}\right)$ of normal fuzzy sets with the induced Hausdorff metric $d_{\infty}$ (see Kloeden and Diamond [8]).
\end{abstract}

Keywords : Fuzzy Dynamical Systems, Global Attractors.

Subjclass: 37B25, 54A40, 54H20. 


\section{Introduction}

To find attractors of a discrete dynamical system on a infinite dimensional space, one is faced with the problem of characterizing the compact subsets of that space. This is a topological issue, and in some sense, one is generally led to the conclusion that there are "few" compact sets in that space [17]. As a consequence one has to rely on properties of regularity of the mapping that generate the dynamical system. There are some concepts affecting the dissipativity of a system that are irrelevant for the finite dimensional spaces but important when the space is infinite dimensional. In our specific case we will focus on the concept of asymptotic smoothness of the mapping on the space of fuzzy vectors.

The concept of asymptotic smoothness was introduced by J. Hale in [10]. Every continuous transformation between finite dimensional vector spaces are asymptotically smooth. This is not true for transformations between infinite dimensional Banach spaces or metric spaces. From the dynamical point of view, an important property of an asymptotically smooth map is that the existence of a compact set that attracts locally points implies that the compact set also attracts locally compact sets. A practical consequence of this result is the use of an iteration procedure to approximate global attractors. J. Hale gives some examples of asymptotic smooth transformations in infinite dimensional spaces. Our objective in this paper is to provide another class of such mappings using the Zadeh's extensions of a continuous transformation in $\mathbf{R}^{n}$.

Extending a map $T: \mathbf{R}^{n} \rightarrow \mathbf{R}^{n}$ to a map in the fuzzy space $\hat{T}: \mathcal{F}\left(\mathbf{R}^{n}\right) \rightarrow \mathcal{F}\left(\mathbf{R}^{n}\right)$ using the extension principle is a standard procedure in fuzzy sets theory [18]. This procedure is particularly useful if one knows a deterministic process but has a fuzzy initial condition, see, for instance [2]. Many works on fuzzy dynamical systems treat the case of Zadeh extensions [9, 12, 13, 6], since they have many suitable properties. For instance, such systems may preserve the $\alpha$-levels.

Results that associate the regularity of a transformation and its Zadeh's extension can be found in the works of Nguyen [14], Barros et al. [1, 16] and Ma et al. [9].

In this paper we study how far the dynamical properties of a map 
in $\mathbf{R}^{n}$ are transported to its Zadeh's extension defined on $\mathcal{F}\left(\mathbf{R}^{n}\right)$ [14]. Also, we indicate a procedure to obtain an attractor, when it exists.

Attractors of a such discrete dynamical system in the space $\mathcal{F}\left(\mathbf{R}^{n}\right)$ appear often as limit sets of an iteration system. Some instances of this application can be found in the works of Cabrelli et al. [4] and Barros et al. [2].

In [4], Cabrelli et al. introduce the iterated fuzzy sets systems, and study their orbits as approximation of fractals and other images. These sets should be global attractors in $\mathcal{F}\left(\mathbf{R}^{n}\right)$, and this forces the family of transformations $T_{k}: \mathcal{F}\left(\mathbf{R}^{n}\right) \rightarrow \mathcal{F}\left(\mathbf{R}^{n}\right)$, that originate the iterated fuzzy sets systems, to be asymptotically smooth. In that paper the hypothesis that $T_{k}$ are Zadeh's extensions of Lipschitzian maps in $\mathbf{R}^{2}$ is sufficient for the existence of a global attractor.

In [2], Barros et al. considers the fuzzification of population growth models. This is obtained considering the Zadeh's extension of the so called Malthus-Verhulst (or logistic) models. The work shows that, besides the known attractors of the deterministic logistic system, there are other fuzzy attractors.

The paper is organized as follows: In section 2 we recall the main definitions and known results from dynamical systems theory and fuzzy set analysis. In section 3 we present a discussion of the properties of compact sets in $\mathcal{F}\left(\mathbf{R}^{n}\right)$ that affect our work. Section 4 contains our main results that give some condition under which the Zadeh's extension of a continuous transformation is asymptotically smooth. In the last section we present some examples.

\section{Preliminaries}

If $X$ is a metric space and $T: X \rightarrow X$ is a continuous transformation then we have a discrete dynamical system. For the basic notations and definitions on dynamical systems we refer to Hale [10]. We say that $T$ is asymptotically smooth if, for each nonempty bounded and closed set $B \subset X$ for which $T(B) \subset B$, there is a compact set $J \subset B$ such that $J$ attracts $B$. We recall that $J$ attracts $B$ if for each neighborhood of $J$ there is a positive $n_{0}$ such that $T^{n}(B)$ is contained in that neighborhood for all $n \geq n_{0}$ (see Hale [10] page 9). An important property is that if $T$ is an asymptotically smooth map then a set 
attracts locally points if and only if it attracts locally compact sets. Cooperman [5] and Brumley [3] have given examples showing that this is not always true for general continuous transformations on infinite dimensional Banach Spaces.

The concepts of limit sets of dynamical systems are classical. Here we will need the following: the $\omega$-limit of a subset $B$ of $X$ is given by:

$$
\omega(B)=\bigcap_{n>0} \operatorname{cl}\left(\bigcup_{k \geq n} T^{k}(B)\right) .
$$

It is clear that if $B$ is such that $T(B) \subset B$ then

$$
\omega(B)=\bigcap_{n>0} \operatorname{cl}\left(T^{n}(B)\right)
$$

The next Lemma can be found in Hale [10] (page 11, Cor. 2.2.4).

Lemma 1 If $\mathrm{T}$ is asymptotically smooth and $B$ is a nonempty bounded set such that its positive orbit is bounded, then $\omega(B)$ is nonempty, compact, and invariant and $\omega(B)$ attracts $B$.

Next we recall some definitions on fuzzy metric spaces.

The family of all compact nonempty subsets of $\mathbf{R}^{n}$ will be denoted as $\mathcal{Q}\left(\mathbf{R}^{n}\right)$. We also indicate $\mathcal{F}\left(\mathbf{R}^{n}\right)$ as the family of fuzzy sets $u$ : $\mathbf{R}^{n} \rightarrow[0,1]$ whose $\alpha$-level:

$$
\begin{gathered}
{[u]^{\alpha}=\left\{x \in \mathbf{R}^{n}: u(x) \geq \alpha\right\} \quad 0<\alpha \leq 1 \text { and }} \\
{[u]^{0}=\operatorname{cl}\left\{x \in \mathbf{R}^{n}: u(x)>0\right\}}
\end{gathered}
$$

are in $\mathcal{Q}\left(\mathbf{R}^{n}\right)$.

It is known that the metric

$$
d_{\infty}(u, v)=\sup _{0 \leq \alpha \leq 1} h\left([u]^{\alpha},[v]^{\alpha}\right)
$$

where $h$ is the Hausdorff metric in $\mathcal{Q}\left(\mathbf{R}^{n}\right)$, makes the spaces $\left(\mathcal{F}\left(\mathbf{R}^{n}\right), d_{\infty}\right)$ into complete metric spaces [15].

Let $f: \mathbf{R}^{n} \rightarrow \mathbf{R}^{n}$ be a mapping then we define the Zadeh's extension as:

$$
\hat{f}(u)(x)=\left\{\begin{array}{lll}
\sup _{\tau \in f^{-1}(x)} u(\tau) & \text { if } & f^{-1}(x) \neq \emptyset \\
0 & \text { if } & f^{-1}(x)=\emptyset
\end{array}\right.
$$


for all fuzzy sets $u$.

The problem we addressed here is: What are the conditions for a continuous transformations on $\mathbf{R}^{n}$ to have its Zadeh's extensions asymptotically smooth? Note that since the Zadeh's extensions of the identity in $\mathbf{R}^{n}$ is the identity in $\mathcal{F}\left(\mathbf{R}^{n}\right)$, and that not all bounded closed set in $\mathcal{F}\left(\mathbf{R}^{n}\right)$ are compact, the identity map doesn't have this property.

The proof of the following results can be found in [1].

Theorem 1 If $f: \mathbf{R}^{n} \rightarrow \mathbf{R}^{n}$ is continuous then $\hat{f}: \mathcal{F}\left(\mathbf{R}^{n}\right) \rightarrow$ $\mathcal{F}\left(\mathbf{R}^{n}\right)$ is well defined and for all $\alpha \in[0,1]$ we have

$$
[\widehat{f}(u)]^{\alpha}=f\left([u]^{\alpha}\right) \text {. }
$$

We will need also a recent result of Roman-Flores et al. [16]

Theorem 2 If $f: \mathbf{R}^{n} \rightarrow \mathbf{R}^{n}$ is continuous then $\hat{f}: \mathcal{F}\left(\mathbf{R}^{n}\right) \rightarrow$ $\mathcal{F}\left(\mathbf{R}^{n}\right)$ is continuous with respect to the metric $d_{\infty}$.

\section{Some facts on compact sets on $\mathcal{F}\left(\mathbf{R}^{n}\right)$}

Our strategy is to study the problem using the definition of an asymptotically smooth map. Then we should be able to determine when a subset of $\mathcal{F}\left(\mathbf{R}^{n}\right)$ is compact or not. In the book of Kloeden and Diamond [8] one finds the characterization of compact sets in the space of fuzzy sets with convex levels which does not fits our purpose since our levels are only compact sets in $\mathbf{R}^{n}$. The best approach we have found in the literature is the article of Roman-Flores [17]. In that paper it is shown how difficult it is to find a compact set in $\mathcal{F}\left(\mathbf{R}^{n}\right)$. A result that is of great importance here is the following: If $K$ is a compact set in $\mathbf{R}^{n}$ then $J_{K}=\left\{u \in \mathcal{F}\left(\mathbf{R}^{n}\right):[u]^{0} \subset K\right\}$ is compact if and only if $K$ has diameter zero! Our candidate to be an attractor will be of this type but we have also good properties for this candidate.

Lemma 2 Let $K \subset \mathbf{R}^{n}$ be a compact set and $A=\left\{u \in \mathcal{F}\left(\mathbf{R}^{n}\right)\right.$ :

$\left.[u]^{0} \subset K\right\}$. Then $A$ is a bounded closed set of the metric space $\left(\mathcal{F}\left(\mathbf{R}^{n}\right), d_{\infty}\right)$. 
Proof: To see that $A$ is bounded note that the distance of $A$ to the point $\hat{0}$ (the characteristic function at 0 ) is finite. Indeed, denoting by $h$ the Hausdorff metric between compact sets

$$
\begin{aligned}
d_{\infty}(\hat{0}, A)= & \inf _{u \in A} d_{\infty}(\hat{0}, u)=\inf _{u \in A} \sup _{\alpha \in[0,1]} h\left(\{0\},[u]^{\alpha}\right) \\
& \leq \sup _{x \in K} d(0, x)=M<\infty
\end{aligned}
$$

Now $A$ is closed. In fact, consider a convergent sequence $u_{n}$ in $A$ with limit $u$, the convergence being in the metric $d_{\infty}$ then we have in particular that $h\left(\left[u_{n}\right]^{0},[u]^{0}\right) \rightarrow 0$ and since $\left[u_{n}\right]^{0} \subset K$ then $[u]^{0} \subset K$ proving that $u$ belongs to $A$. In other words $A$ contains all its cluster points and then is closed.

$Q E D$

\section{The asymptotic smoothness problem}

The main results will follow from a sequence of lemmas. Let $f: \mathbf{R}^{n} \rightarrow$ $\mathbf{R}^{n}$ be a continuous transformation and $\hat{f}: \mathcal{F}\left(\mathbf{R}^{n}\right) \rightarrow \mathcal{F}\left(\mathbf{R}^{n}\right)$ its Zadeh's extension which is continuous according Theorem 2. To prove that $\hat{f}$ is asymptotically smooth we have to prove that for each closed bounded set $B \subset \mathcal{F}\left(\mathbf{R}^{n}\right)$ such that $\hat{f}(B) \subset B$ there is a compact set $J \subset B$ that attracts $B$. We take a closed bounded nonempty $B$. Note that for each $u \in \hat{f}(B)$ and $v \in B$ such that $\hat{f}(v)=u$ we have $[u]^{\alpha}=[\widehat{f}(u)]^{\alpha}=f\left([u]^{\alpha}\right)$, by using Theorem 1 .

Lemma 3 Let $B$ be as above and define $B_{\alpha}=\operatorname{cl}\left(\bigcup_{u \in B} \alpha\right) \subset \mathbf{R}^{n}$. There exists a compact set $K \subset \mathbf{R}^{n}$ such that $[u]^{0} \subset K$ for all $u \in B$. Hence $B_{\alpha}$ is bounded for each $\alpha \in[0,1]$.

Proof: Take a point $x$ in $\mathbf{R}^{n}$ and denote as $\hat{x}$ its characteristic function. Since $B$ is bounded there exists a $r>0$ such that the ball with center in $\hat{x}$ and radius $r$ contains the entire set $B$. In other words, for all $u \in B d_{\infty}(\hat{x}, u) \leq r$. According to a result that can be found in [1] this metric can be written as

$$
d_{\infty}(\hat{x}, u)=\sup _{0 \leq \alpha \leq 1} \inf \left\{a:[u]^{\alpha} \subset B_{a}(x) \text { and } x \in B_{a}\left([u]^{\alpha}\right)\right\}
$$


where $B_{a}(x)$ denotes the Euclidean ball centered in $x$. Then it follows immediately that $[u]^{0} \subset B_{r}(x) \subset \operatorname{cl} B_{r}(x)=K$. Now since $[u]^{\alpha} \subset[u]^{0}$ follows $[u]^{\alpha} \subset K$ what proves the lemma.

Lemma 4 Consider $B_{\alpha}$ as in Lemma 3. Then $B_{\alpha}$ are closed bounded and satisfy $f\left(B_{\alpha}\right) \subset B_{\alpha}$. Therefore there is a compact set $J_{\alpha} \subset B_{\alpha}$ that attracts $B_{\alpha}$.

Proof: In fact the only assertion that has to be proved is that $f\left(B_{\alpha}\right) \subset$ $B_{\alpha}$. The rest follows immediately from definitions, Lemma 2 and the fact that every continuous transformation in $\mathbf{R}^{n}$ is asymptotically smooth.

Take $x$ in $B_{\alpha}$. By definition $x$ is the limit of a sequence $x_{n}$ with $x_{n} \in\left[u_{n}\right]^{\alpha}$ and $u_{n} \in B$. Therefore we have

$$
f\left(x_{n}\right) \in f\left(\left[u_{n}\right]^{\alpha}\right)=[\widehat{f}(u)]^{\alpha} \subset B_{\alpha} .
$$

Since $f$ is continuous $f\left(x_{n}\right)$ converges to $f(x) \in B_{\alpha}$ and this completes this proof.

Using the Lemma 1 we can define the special compact invariant sets $J_{\alpha}=\omega\left(B_{\alpha}\right)$. These are the attractors we will consider. Now we can prove.

Lemma 5 For $0 \leq \alpha_{1} \leq \alpha_{2} \leq 1$ we have $J_{\alpha_{2}} \subset J_{\alpha_{1}}$.

Proof: We have that $B_{\alpha_{2}} \subset B_{\alpha_{1}}$. Observing that in this case the omega limits can be written as:

$$
J_{\alpha_{2}}=\omega\left(B_{\alpha_{2}}\right)=\bigcap_{n \geq 0} f^{n}\left(B_{\alpha_{2}}\right) \subset \bigcap_{n \geq 0} f^{n}\left(B_{\alpha_{1}}\right)=J_{\alpha_{1}}
$$

and then follows the result.

We define $J=\left\{u \in B:[u]^{0} \subset J_{0}\right\}$.

Theorem 3 If $\widehat{f}: \mathcal{F}\left(\mathbf{R}^{n}\right) \rightarrow \mathcal{F}\left(\mathbf{R}^{n}\right)$ is asymptotically smooth then $J$ is nonempty for all nonempty closed bounded $B$ with $\hat{f}(B) \subset B$. 
Proof: We take a closed bounded $B$ with $\hat{f}(B) \subset B$. Since we are assuming that $\hat{f}$ is asymptotically smooth, we can use Lemma 1 to construct the attractor $K=\omega(B)$ which is also nonempty, compact and invariant. Again, take $K_{0}=\mathrm{cl}_{u \in K}[u]^{0}$. It's easy to see that

a) $K_{0}$ is a compact set contained in $B_{0}$. And this is clear.

b) $K_{0}$ is an invariant set for $f$ (i.e. $\left.f\left(K_{0}\right)=K_{0}\right)$. In fact, if $x \in K_{0}$ then we know that $x$ is a limit of a sequence $x_{n}$ such that $x_{n} \in\left[u_{n}\right]^{0}$ and $u_{n} \in K$ then follows: $f(x)=\lim _{n \rightarrow \infty} f\left(x_{n}\right)$. Now

$$
f\left(x_{n}\right) \in f\left(\left[u_{n}\right]^{0}\right)=\left[\hat{f}\left(u_{n}\right)\right]^{0} \in K_{0}
$$

this shows that $f\left(K_{0}\right) \subset K_{0}$.

To prove that $K_{0} \subset f\left(K_{0}\right)$, we repeat the process taking $x \in K_{0}$ and $x_{n}$ as above. Now since $K$ is invariant for $\hat{f}$ we have that $u_{n}=$ $\hat{f}\left(v_{n}\right)$ where $v_{n} \in K$. Then for each $n \geq 0$ we have $x_{n}=f\left(y_{n}\right)$ where $y_{n} \in\left[v_{n}\right]^{0} \subset K_{0}$. Choosing a subsequence, if necessary, we take $y=\lim _{n \rightarrow \infty} y_{n}$. By continuity of $f$ follows that $x=f(y)$. Hence $K_{0}$ is an invariant set of $f$.

Now $J_{0}$ attracts $B_{0}$ and also $K_{0}$. This means that for each $\epsilon \geq 0$ there is an $n_{0}$ such that for $n \geq n_{0}, f^{n}\left(K_{0}\right) \subset N\left(J_{0}, \epsilon\right)$ or $K_{0} \subset$ $N\left(J_{0}, \epsilon\right)$ using the invariance. Here $N\left(J_{0}, \epsilon\right)$ stands for an $\epsilon$-neighborhood of $J_{0}$. This proves that in fact $K_{0} \subset J_{0}$ because $\epsilon$ is arbitrary and then $J \neq \emptyset$.

In particular if $J=\emptyset$ then $\hat{f}$ isn't asymptotically smooth.

QED.

Theorem 4 Suppose that $\hat{f}: \mathcal{F}\left(\mathbf{R}^{n}\right) \rightarrow \mathcal{F}\left(\mathbf{R}^{n}\right)$ is asymptotically smooth, $B$ a nonempty, bounded, closed subset of $\mathcal{F}\left(\mathbf{R}^{n}\right)$ such that $\hat{f}(B) \subset B, K=\omega(B)$ and $J_{\alpha}=\omega\left(B_{\alpha}\right)$. Then $K_{\alpha}=J_{\alpha}$.

Proof: The proof that $K_{\alpha} \subset J_{\alpha}$ can be done as in the above Theorem changing the index 0 by $\alpha$. It remains to prove that $J_{\alpha} \subset K_{\alpha}$. We know that $K$ attracts $B$, therefore given $\epsilon>0$ there exists $n_{0}$ such that for $n \geq n_{0}, \hat{f}^{n}(B) \subset N(K, \epsilon)$ this implies that for each $\alpha \in[0,1]$, $f^{n}\left(B_{\alpha}\right) \subset N^{*}\left(K_{\alpha}, \epsilon\right)$ where

$$
N^{*}\left(K_{\alpha}, \epsilon\right)=\left\{x \in \mathbf{R}^{n}: d\left(x, K_{\alpha}\right) \leq \epsilon\right\} .
$$


It follows from the definition of $J_{\alpha}$ that

$$
J_{\alpha} \subset \bigcap_{n \geq n_{0}} f^{n}\left(B_{\alpha}\right) \subset N^{*}\left(K_{\alpha}, \epsilon\right)
$$

This is true for every $\epsilon>0$ then follows the result.

QED.

The set $J$, according to the above proposition, is the best candidate in which we can find an attractor. It could be the empty set, and in this case the Zadeh's Extension isn't asymptotically smooth. We give some examples where we can decide on the smoothness of the transformation and even determine the attractors.

\section{Examples}

We present two examples.

Let $f: \mathbf{R}^{n} \rightarrow \mathbf{R}^{n}$ be a continuous transformation, such that $\{0\}$ is the unique global attractor of $f$. Then $\hat{f}: \mathcal{F}\left(\mathbf{R}^{n}\right) \rightarrow \mathcal{F}\left(\mathbf{R}^{n}\right)$ is asymptotically smooth.

It is clear that $\hat{f}: \mathcal{F}\left(\mathbf{R}^{n}\right) \rightarrow \mathcal{F}\left(\mathbf{R}^{n}\right)$ is continuous. We take a bounded closed set $B \in \mathcal{F}\left(\mathbf{R}^{n}\right)$ such that $\hat{f}(B) \subset B$. Now we shall prove that:

(A) $\chi_{\{0\}}$ attracts $B$;

(B) $\chi_{\{0\}} \subset B$.

Since the set $\left\{\chi_{\{0\}}\right\}$ is compact in $\mathcal{F}\left(\mathbf{R}^{n}\right)$ it follows our result.

To prove (A) note that for each $\alpha \in[0,1]$ the set $B_{\alpha}=\operatorname{cl}\left(\bigcup_{u \in B}[u]^{\alpha}\right) \subset$ $\mathbf{R}^{n}$ is compact, and then $\{0\}$ attracts $B_{\alpha}$. This means that for each $\varepsilon>0$ there is an $n_{\alpha} \in \mathbf{N}$ such that $f^{n}\left(B_{\alpha}\right) \subset N(\{0\}, \varepsilon)$ for all $n>n_{\alpha}$. Here $N(\{0\}, \varepsilon)$ denote the $\varepsilon$-neighborhood of $\{0\}$. But since $B_{\alpha} \subset B_{0}$ we have $f^{n}\left(B_{\alpha}\right) \subset N(\{0\}, \varepsilon)$ for all $n>n_{0}$.

For each $u \in B$, it follows that $f^{n}\left([u]^{\alpha}\right) \subset N(\{0\}, \varepsilon)$ and since $f$ is continuous $\left[\hat{f}^{n}(u)\right]^{\alpha} \subset N(\{0\}, \varepsilon)$. From this it follows the assertion (A).

For the item $(\mathrm{B})$ we take $n_{0}$ such that $d_{\infty}\left(\hat{f}^{n}(B), \chi_{\{0\}}\right) \leq \varepsilon$ for $n>n_{0}$. This implies that $\chi_{\{0\}}$ is in a $\varepsilon$-neighborhood of $\hat{f}^{n}(B)$ and then in a $\varepsilon$-neighborhood of $B$, because $\hat{f}^{n}(B) \subset B$. As this last assertion is true for any $\varepsilon>0$ we must have $\chi_{\{0\}} \in B$. The proof of the result is complete. 
As a particular case, we can take $f(x)=A x$ where $A$ is a linear operator. We can restrict the analysis to the eigenspace associated to the eigenvalues whose absolute values are less than one, and the above result applies.

The next example shows that it is not always true that the Zadeh's extension is asymptotically smooth.

If $f: \mathbf{R}^{n} \rightarrow \mathbf{R}^{n}$ has a compact set $K$ with infinite points as attractor, then one can easily see that the set

$$
B=\left\{u \in \mathcal{F}\left(\mathbf{R}^{n}\right):[u]^{0} \subset K\right\}
$$

is a bounded closed set for which $\hat{f}(B)=B$. Since $B$ is not compact, according to Roman-Flores [17], then $\hat{f}$ is not asymptotically smooth. The meaning of this last example is that if $\hat{f}$ has a global attractor it will be hard to detect it since we can not use the theory for asymptotically smooth transformation.

Theorem 4 can be understood as a way to produce fuzzy attractors in a levelwise manner, although there is yet the problem to find out a condition which decides if this attractor certainly exists. Another possible way to follow, is to consider the dissipativity and asymptotic smoothness of time continuous dynamical systems in the sense of Hüllermeier [11]. For this case, the concept of attractor has to be generalized as in Diamond [7].

\section{References}

[1] L. C. Barros, R. C. Bassanezi and P. A. Tonelli - "On the continuity of Zadeh's extension" - Proceedings Seventh IFSA World Congress, Prague, Vol. II, pp. 3-8, (1997).

[2] L. C. Barros, R. C. Bassanezi and P. A. Tonelli - "Fuzzy modeling in populations dynamics" - Ecological Modeling- 128, pp. 27-33 (2000). 
[3] W. E. Brumley - " On the asymptotic behavior of solutions of differential difference equations of neutral type" - J. of Differential Equations 7, pp. 175-188 (1970).

[4] C. A. Cabrelli, B. Forte, U. Molter and E. Vrscay - "Iterated Fuzzy Sets Systems: A new approach to the inverse for fractals and other sets" - J. of Math. Anal. and Appl. 171, pp. 79-100 (1992).

[5] G. Cooperman - " $\alpha$-Condensing maps and dissipative processes" - Ph. D. Thesis, Brown University, Providence, R. I. (1978).

[6] P. Diamond - "Chaos in iterated fuzzy systems" - J. of Mathematical Analysis and Applications- 184, pp. 472-484 (1994).

[7] P. Diamond - "Time Dependent Differential Inclusions, Cocycle Attractors and Fuzzy Differential Equations" , IEEE Trans. On Fuzzy Systems - Vol. 7, pp. 734-740. (1999).

[8] P. Diamond and P. Kloeden - "Metric Spaces of Fuzzy Sets: Theory and Applications" - World Scientific Pub. (1994).

[9] M. Friedmann, M. Ma and A. Kandel - "Numerical solutions of fuzzy differential and integral equations" - Fuzzy Sets and Systems 106, pp. 35-48 (1999).

[10] J. K. Hale - "Asymptotic Behavior of Dissipative Systems"- Math. Surveys and Monographs 25, American Mathematical Society, Providence (1988).

[11] E. Hüllermeier "An Approach to Modeling and Simulation of Uncertain Dynamical Systems"-Int. J. Uncertainty, Fuzziness, Knowledge-Bases Syst. Vol. 5, pp. 117-137 (1997).

[12] P. E. Kloeden - "Fuzzy dynamical systems" - Fuzzy Sets and Systems- 7, pp. 275-296 (1982).

[13] P. E. Kloeden - "Chaotic iterations of fuzzy sets" - Fuzzy Sets and Systems- 42, pp. 37-42 (1991). 
[14] H. T. Nguyen - "A note on the extension principle for fuzzy sets" - J. Math. Anal. Appl. 64, pp. 369-380 (1978).

[15] M. L. Puri and D. A. Ralescu - "Fuzzy Random Variables" J. of Mathematical Analysis and Applications- 114, pp. 409-422 (1986).

[16] H. Roman-Flores, L. C. Barros and Bassanezzi, R. - "A note on Zadeh's Extensions" - Fuzzy Sets and Systems-117, pp. 327-331 (2001).

[17] H. Roman-Flores - "On the Compactness of $E(X)$ " - Appl. Math. Lett. 11, pp. 13-17. (1998).

[18] L. A. Zadeh - "Fuzzy sets" - Inform. Control- 8, pp. 338-353 (1965).

Received : May, 2002.

\section{Laécio C. Barros}

Departamento de Matemática Aplicada

Universidade estadual de Campinas

13081-970 CP 6065, Campinas

Brasil

e-mail : laeciocb@ime.unicamp.br

\section{Suzana A. Oliveira Souza}

Departamento de Matemática Aplicada

Universidade de São Paulo

05508-900 CP 66281

São Paulo

Brasil

e-mail : suzanabreu@uol.com.br

and 


\section{Pedro A. Tonelli}

Departamento de Matemática Aplicada

Universidade de So Paulo

CP 66.281

CEP 05311 - 970

São Paulo

Brasil

e-mail : tonelli@ime.usp.br 